\title{
FRAGMENTATION ENERGY
}

\author{
JEAN BERTOIN, ${ }^{*}$ Université Paris 6 and Institut Universitaire de France \\ SERVET MARTÍNEZ, ${ }^{* *}$ Universidad de Chile, Santiago
}

\begin{abstract}
Motivated by a problem arising in the mining industry, we estimate the energy $\mathscr{E}(\eta)$ that is needed to reduce a unit mass to fragments of size at most $\eta$ in a fragmentation process, when $\eta \rightarrow 0$. We assume that the energy used in the instantaneous dislocation of a block of size $s$ into a set of fragments $\left(s_{1}, s_{2}, \ldots\right)$ is $s^{\beta} \varphi\left(s_{1} / s, s_{2} / s, \ldots\right)$, where $\varphi$ is some cost function and $\beta$ a positive parameter. Roughly, our main result shows that if $\alpha>0$ is the Malthusian parameter of an underlying Crump-Mode-Jagers branching process (with $\alpha=1$ when the fragmentation is mass-conservative), then there exists a $c \in(0, \infty)$ such that $\mathscr{E}(\eta) \sim c \eta^{\beta-\alpha}$ when $\beta<\alpha$. We also obtain a limit theorem for the empirical distribution of fragments of size less than $\eta$ that result from the process. In the discrete setting, the approach relies on results of Nerman for general branching processes; the continuous approach follows by considering discrete skeletons. In the continuous setting, we also provide a direct approach that circumvents restrictions induced by the discretization.
\end{abstract}

Keywords: Fragmentation; general branching process, energy

2000 Mathematics Subject Classification: Primary 60J80

Secondary 60F15

\section{Introduction}

One of the main goals in the mining industry is to extract blocks of metallic ore and then separate the metal from the valueless material. To do so, fragmentation is carried out in a series of steps, the first one being blasting, after which the material is transported to crushers, grinders, and, finally, to mills. At each step, the particles are screened and, if they are smaller than the diameter of the mesh of a classifying grid, they proceed to the next step. The process finishes when the material attains a size sufficiently small for mining purposes (more precisely, to enable efficient physicochemical processing). In the blasting and crushing processes, output sizes are known not to be optimal in terms of the global energy cost. Obviously, one of the problems that faces the mining industry is that of minimizing the energy used in these processes.

To get an idea of the magnitudes involved in fragmentation in the mining industry, at Chuquicamata (the largest open-pit copper-mine on earth), located in northern Chile, nearly 600000 tonnes of material are blasted and transported per day. Out of this, nearly 200000 tonnes are defined as mineral; the rest is waste or sterile. The average copper content of the mineral is around $1 \%$. After blasting, there are two methods of mechanically decreasing the mineral size. The conventional process uses three types of crusher (primary, secondary, and tertiary) and the particles are forwarded to the mills once they are of size less than $\frac{1}{2}$ in. The mills

Received 25 August 2004; revision received 8 December 2004.

* Postal address: Laboratoire de Probabilités et Modèles Aléatoires, Université Paris 6, 175, rue de Chevaleret, F-75013

Paris, France. Email address: jbe@ccr.jussieu.fr

** Postal address: CMM-DIM, Universidad de Chile, Casilla 170-3 Correo 3 Santiago, Chile.

Email address: smartine@dim.uchile.cl 
use two types of breakage media: steel bars and balls. The fragmentation process is stopped once the particles have a size less than $2 \times 10^{-5} \mathrm{~m}$. In the other process, semiautogenous mills are fed with particles of size less than $8 \mathrm{in}$. with a similar end product of size $2 \times 10^{-5} \mathrm{~m}$.

In this work, we consider discrete and continuous models of fragmentation processes in which particles break independently of each other and in a self-similar way (see Sections 2 and 3 for precise statements of our assumptions). The self-similarity hypothesis agrees with observations made in the mining industry; see, e.g. [9], [12], [13], and [16]. We assume that the energy required to break a block of size $s$ into a set of smaller blocks of sizes $\left(s_{1}, s_{2}, \ldots\right.$ ) (in one unit of time in the discrete case, and instantaneously in the continuous case) is of the form $s^{\beta} \varphi\left(s_{1} / s, s_{2} / s, \ldots\right)$, where $\varphi$ is a cost function and $\beta>0$ a fixed parameter. For generality, we do not require $\varphi$ to be nonnegative, although this would be physically relevant.

When $\varphi\left(s_{1}, s_{2}, \ldots\right)=\sum_{n=1}^{\infty} s_{n}^{\beta}-1$, the energy (which is always nonnegative whenever $\beta<1$ and $\sum_{n=1}^{\infty} s_{n}=1$ ) is called a potential because the total energy needed to break a series of masses $\left(m_{1}, m_{2}, \ldots\right)$, summing to 1 , into a finer distribution $\left(m_{1}^{\prime}, m_{2}^{\prime}, \ldots\right)$ is given by $\sum_{i} m_{i}^{\prime \beta}-\sum_{j} m_{j}^{\beta}$, and this is independent of the fragmentation path leading from the initial configuration to the final one. In this case, if $\left\{x_{i}\right\}$ is the set of the sizes of the fragments obtained by removing each fragment from the process at the time when it becomes less than a certain bound $\eta$, then the energy needed to attain this state, starting from the unit mass, is $\varepsilon(\eta)=\sum_{i} x_{i}^{\beta}-1$. This corresponds to the laws of Charles [8], Walker et al. [15], and Bond [7]: $\mathrm{d} E(r)=C \mathrm{~d} r / r^{\gamma}$, where $\mathrm{d} E(r)$ is the specific energy needed to change the size of a particle from $r$ to $r-\mathrm{d} r$. The potential energy model is one of those most widely used in mining.

In Section 2, we will deal with the discrete setting. Our approach to the study of the energy problem relies on the formulation of the fragmentation chain as a general (i.e. Crump-ModeJagers) branching process in which the time parameter corresponds to the logarithm of the sizes of the fragments. This formulation enables us to apply theorems of Nerman [11], from which estimates for the energy follow. The same technique also yields interesting limit theorems for the empirical distribution of the fragments of size less than $\eta$, when suitably normalized. In Section 3, the continuous setting is treated first by considering a discrete skeleton and estimating errors, and, second, by taking a direct approach, based on moment estimates via renewal theory for conservative fragmentations, that circumvents some restrictions imposed on the exponent $\beta$ by the discretization technique. Finally, in Section 4, we briefly derive conclusions of our study.

In what follows, we shall implicitly assume that the process starts from a single block with unit size, except when otherwise indicated.

\section{Discrete framework}

In this section, we consider the following simple setting, in which the system has a discrete genealogical structure. Specifically, let us denote by

$$
\ell:=\bigcup_{n=0,1, \ldots} \mathbb{N}^{n}
$$

the infinite regular tree, with the usual convention that $\mathbb{N}^{0}=\{\varnothing\}$. We will often refer to $\ell$ as the genealogical tree; its elements are called individuals. We think of $\varnothing$ as the ancestor and, for every $i=\left(i_{1}, \ldots, i_{n}\right) \in \ell$ and $j \in \mathbb{N}$, of $i j:=\left(i_{1}, \ldots, i_{n}, j\right)$ as the $j$ th child of the individual $i$. For each $i=\left(i_{1}, \ldots, i_{n}\right) \in \ell$, we call $n$ the generation of $i$ and write $|i|=n$, with the obvious convention $|\varnothing|=0$. 
Individuals are used to label the fragments produced by a fragmentation chain; we mark each individual $i \in \ell$ with a real number $\sigma_{i}$, where $\mathrm{e}^{-\sigma_{i}}$ is the size of the fragment with label $i$. In other words, the marks assigned to the individuals are given by minus the logarithm of the size of the corresponding fragments. More precisely, recall that we start from a single block of unit size, and denote the sequence of the sizes of the fragments resulting from the dislocation of this block by $\left(s_{1}, s_{2}, \ldots\right)$. We then assign the mark $\sigma_{\varnothing}=0$ to the ancestor and $\sigma_{i}=-\log s_{i}$ to each individual $i \in \mathbb{N}^{1}$ of the first generation. Marks are assigned similarly to the next generations.

Let us now specify the dynamics. We assume that each fragment of size $s>0$ breaks into a sequence of smaller fragments of sizes $s_{1}, s_{2}, \ldots$, where the sequence of ratios $s_{1} / s, s_{2} / s, \ldots$. has a fixed distribution $v$ (i.e. $v$ does not depend on the size $s$ ). Hence, $v$ is a probability measure on $[0,1]^{\mathbb{N}}$, which will be referred to as the dislocation law. We shall always implicitly assume that the dislocation law is not geometric, in the sense that there is no real number $r \in(0,1)$ such that $s_{n} \in\left\{1, r, r^{2}, \ldots\right\}$ for all $n$, almost surely (a.s.).

The fragmentation is called dissipative if $\sum_{n=1}^{\infty} s_{n} \leq 1 v$-a.s. and the inequality is strict with positive probability (this occurs, for instance, when size is equivalent to mass and some dust, i.e. infinitesimal fragments, can be produced). It is called conservative if $\sum_{n=1}^{\infty} s_{n}=1 v$-a.s. Note that the model also includes the case in which $\sum_{n=1}^{\infty} s_{n} \geq 1$ with positive probability, which occurs, for instance, when by the size of a fragment we mean its diameter.

Finally, we assume that different fragments evolve independently. In other words, the measure-valued process

$$
Z^{n}(\mathrm{~d} x)=\sum_{i \in \mathbb{N}^{n}} \delta_{\sigma_{i}}(\mathrm{~d} x), \quad x \in[0, \infty),
$$

where $\delta_{\sigma_{i}}$ denotes the Dirac point mass at $\sigma_{i}$, is a branching random walk, in the sense of [6], whose reproduction intensity is given, in terms of the dislocation law, by

$$
\mathrm{E}\left(\left\langle Z^{1}, f\right\rangle\right)=\int_{[0,1]^{\mathbb{N}}} \sum_{n=1}^{\infty} f\left(-\log s_{n}\right) v(\mathrm{~d} \mathfrak{s}),
$$

where $\mathfrak{s}=\left(s_{n}, n \in \mathbb{N}\right)$ denotes a generic sequence in $[0,1]$ and $f:[0, \infty) \rightarrow \mathbb{R}$ a generic bounded, measurable function. We stress that the random point measure $Z^{n}$ does not take into account the fragments of size 0 (i.e. those for which $\sigma_{i}=\infty$ ).

Throughout this section, we shall assume that the Malthusian hypothesis is satisfied; that is, that there exists an $\alpha>0$, called the Malthusian parameter, such that

$$
\mathrm{E}\left(\left\langle Z^{1}, \mathrm{e}^{-\alpha \cdot}\right\rangle\right)=\int_{[0,1]^{\mathbb{N}}} \sum_{n=1}^{\infty} s_{n}^{\alpha} v(\mathrm{~d} \mathfrak{s})=1 ;
$$

and that the $L \log L$ condition holds, where, for every $x \geq 0, \log ^{+} x=\log (x \vee 1)$ :

$$
\mathrm{E}\left(\left\langle Z^{1}, \mathrm{e}^{-\alpha \cdot}\right\rangle \log ^{+}\left\langle Z^{1}, \mathrm{e}^{-\alpha \cdot}\right\rangle\right)=\int_{[0,1]^{\mathbb{N}}} \sum_{n=1}^{\infty} s_{n}^{\alpha} \log ^{+}\left(\sum_{m=1}^{\infty} s_{m}^{\alpha}\right) v(\mathrm{~d} \mathfrak{s})<\infty .
$$

Then, it is well known (see [6]) that the process

$$
W_{n}:=\left\langle Z^{n}, \mathrm{e}^{-\alpha \cdot}\right\rangle=\sum_{i \in \mathbb{N}^{n}} \mathrm{e}^{-\alpha \sigma_{i}}
$$


is a uniformly integrable martingale that converges a.s. and in $L^{1}(\mathrm{P})$ to $W_{\infty}$, and that $W_{\infty}>0$ a.s. conditionally on nonextinction (i.e. on $Z^{n} \neq 0$ for all $n$ ). Observe that, in the conservative case, the Malthusian parameter is $\alpha=1$ and the martingale $W_{n}$ is constant (in $n$ ).

Now recall, from the introduction, the problem that motivates this work. Fix $\eta>0$ and let the process evolve until every fragment in the system has size less than $\eta$, in the sense that each fragment of size less than $\eta$ is instantaneously frozen in the system. Suppose that, at each step, the energy cost of the dislocation of a block of size $s$, producing a sequence of fragments of sizes $s_{1}, s_{2}, \ldots$, is $s^{\beta} \varphi\left(s_{1} / s, s_{2} / s, \ldots\right)$, where $\beta$ is a positive parameter and $\varphi:[0,1]^{\mathbb{N}} \rightarrow \mathbb{R}$ a measurable symmetric function (the cost function). The total energy cost of the process, stopped when all the fragments have size less than $\eta$, is thus given by

$$
\mathcal{E}(\eta):=\sum_{i \in \ell} \mathbf{1}_{\left\{\sigma_{i} \leq-\log \eta\right\}} \mathrm{e}^{-\beta \sigma_{i}} \varphi\left(\Delta_{i}\right),
$$

where $\Delta_{i}=\left(\exp \left(\sigma_{i}-\sigma_{i, n}\right), n \in \mathbb{N}\right)$ is the sequence of ratios of the sizes of the fragments labelled by the children of the individual $i$ to the size of the fragment from which they came, labelled by individual $i$ itself.

We are now able to state the main result in the discrete setting.

Theorem 1. Suppose that $\beta<\alpha$ and $\int_{[0,1]^{\mathbb{N}}}|\varphi(\mathfrak{s})| v(\mathrm{~d} \mathfrak{s})<\infty$, and set

$$
m(\alpha)=\int_{[0,1]^{\mathbb{N}}} \sum_{n=1}^{\infty} s_{n}^{\alpha} \log \left(\frac{1}{s_{n}}\right) v(\mathrm{~d} \mathfrak{s}) .
$$

Then, under the preceding assumptions,

$$
\lim _{\eta \rightarrow 0} \eta^{\alpha-\beta} \mathcal{E}(\eta)=\frac{W_{\infty}}{(\alpha-\beta) m(\alpha)} \int_{[0,1]^{\mathbb{N}}} \varphi(\mathfrak{s}) \nu(\mathrm{d} \mathfrak{s}) \text { in } L^{1}(\mathrm{P}) .
$$

Proof. The idea of the proof is to express the energy functional in terms of a Crump-ModeJagers branching process that arises naturally in this setting, and then to apply convergence results of Nerman [11] to the latter.

We think of the mark $\sigma_{i}$, which has been assigned to each individual $i$ of the genealogical tree $\ell$, as a birth time. Every individual born has an infinite lifetime, and gives birth to children according to a random point process $\xi_{i}$. More precisely, if $s_{1} \geq s_{2} \geq \cdots$ denote the elements of the sequence when ranked in decreasing order of the size of the fragments resulting from the first dislocation of a block of size $s$ (recall that we assume that $s \geq s_{1}$, i.e. dislocations always produce fragments of smaller sizes), then the $n$th child of that individual is born at time $-\log s_{n}$.

The particle system that is obtained in this way is then a general branching process with reproduction intensity

$$
\mu(t):=\mathrm{E}\left(\xi_{\varnothing}([0, t])\right)=\int_{[0,1]^{\mathbb{N}}} \sum_{n=1}^{\infty} \mathbf{1}_{\left\{s_{n} \geq \mathrm{e}^{-t}\right\}} v(\mathrm{~d} \mathfrak{s}), \quad t \geq 0 .
$$

Note that if we index this particle system by generations instead of time, we clearly recover the branching random walk $Z^{n}$. In this setting, the martingale $W_{n}$ coincides with the so-called intrinsic martingale, and if we introduce

$$
Y_{t}:=\sum_{i \in \ell(t)} \mathrm{e}^{-\alpha \sigma_{i}}, \quad t \geq 0,
$$


where $\ell(t)$ denotes the stopping line composed of those individuals, born after time $t$, whose parents are born before or at time $t$, then $\left(Y_{t}, t \geq 0\right)$ is a uniformly integrable martingale with terminal value $W_{\infty}$; see [10].

Next, consider a block of size $s$, and let $\left(s_{1}, s_{2}, \ldots\right)$ be the ranked sequence of the sizes of the fragments that result from its first dislocation. This corresponds to an individual $i$, born at time $\sigma_{i}=-\log s$, that has offspring described by the point process

$$
\xi_{i}([0, t])=\operatorname{card}\left\{n \in \mathbb{N}: s_{n} \geq \mathrm{e}^{-t}\right\} .
$$

For every $t \geq 0$, we set

$$
\phi_{i}(t)=\mathrm{e}^{\beta t} \varphi\left(s_{1} / s, s_{2} / s, \ldots\right),
$$

with $\phi_{i}(t)=0$ for $t<0$. Note that the energy cost of the dislocation is $\mathrm{e}^{-\beta \sigma_{i}} \phi_{i}(0)$ and, hence,

$$
\eta^{-\beta} \mathcal{E}(\eta)=\sum_{i \in \ell} \phi_{i}\left(t-\sigma_{i}\right), \quad t=-\log \eta .
$$

According to Theorem 3.1 and Corollary 3.3 of [11], we have

$$
\lim _{t \rightarrow \infty} \mathrm{e}^{-\alpha t} \sum_{i \in \ell} \phi_{i}\left(t-\sigma_{i}\right)=m_{\infty}^{\phi} W_{\infty} \quad \text { in } L^{1}(\mathrm{P}),
$$

where

$$
m_{\infty}^{\phi}=\frac{\int_{0}^{\infty} \mathrm{e}^{-\alpha t} \mathrm{E}\left(\phi_{\varnothing}(t)\right) \mathrm{d} t}{\int_{0}^{\infty} t \mathrm{e}^{-\alpha t} \mu(\mathrm{d} t)}
$$

see [11, Proposition 2.2, Equation (2.7)]. Calculations immediately yield

$$
m_{\infty}^{\phi}=\frac{\int_{[0,1]^{\mathbb{N}}} \varphi(\mathfrak{s}) v(\mathrm{~d} \mathfrak{s})}{(\alpha-\beta) m(\alpha)}
$$

Putting the pieces together, this completes the proof.

Remark 1. (a) In the case that $\beta>\alpha$, the total energy needed to reduce a block entirely to dust is $\varepsilon(0+)<\infty$ a.s. Indeed, the branching property implies that

$$
\mathrm{E}(\mathscr{E}(0+))=\sum_{n=0}^{\infty} \sum_{|i|=n} \mathrm{E}\left(\mathrm{e}^{-\beta \sigma_{i}} \varphi\left(\Delta_{i}\right)\right)=c \sum_{n=0}^{\infty} \sum_{|i|=n} \mathrm{E}\left(\mathrm{e}^{-\beta \sigma_{i}}\right),
$$

where $c=\int_{[0,1]^{\mathbb{N}}} \varphi(\mathfrak{s}) v(\mathrm{~d} \mathfrak{s})<\infty$. However, the branching property also yields

$$
\sum_{|i|=n} \mathrm{E}\left(\mathrm{e}^{-\beta \sigma_{i}}\right)=\left(\int_{[0,1]^{\mathbb{N}}}\left(\sum_{j=1}^{\infty} s_{j}^{\beta}\right) v(\mathrm{~d} \mathfrak{s})\right)^{n}
$$

Since $\beta>\alpha$, we have $\int_{[0,1]^{\mathbb{N}}}\left(\sum_{j=1}^{\infty} s_{j}^{\beta}\right) v(\mathrm{~d} \mathfrak{s})<1$ and, so, $\mathrm{E}(\mathcal{E}(0+))<\infty$.

(b) Under a rather mild condition on the dislocation law, we can strengthen Theorem 1 to obtain almost-sure convergence; see Condition 5.1 and Theorem 5.4 of [11]. 
(c) In a general branching process, an individual $i$ may be endowed with a life career $\omega_{i}$ that contains, in particular, the information on the ages at which the individual begets its children, but which may have a much richer structure (the crucial hypothesis is that the life careers of different individuals are independent and identically distributed). Theorem 3.1 of [11], which provides the key to our Theorem 1, can be extended to this more general setting; see Theorem 7.3 of [10]. This allows us to extend Theorem 1 to the more general situation in which the energy cost of fragmentation during one unit of time does not only depend on the size of the initial block and that of the resulting fragments, but also on further information given by the life career $\omega_{i}$ of the corresponding individual $i$. In fact, the energy used to fragment a block of size $m$ with life career $\omega_{i}$ is $m^{\beta} \varphi\left(\omega_{i}\right)$, where the cost function $\varphi$ now depends on the whole life career. In this case, Theorem 1 still holds if we replace $\int_{[0,1]^{\mathbb{N}}} \varphi(\mathfrak{s}) v(\mathrm{~d} \mathfrak{s})$ with $\mathrm{E}\left(\varphi\left(\omega_{\varnothing}\right)\right)$. This immediate generalization will play a crucial role in the next section, where we shall tackle the continuous framework using discretization.

Theorem 1 yields interesting asymptotics for the empirical distribution of the fragments resulting from the process stopped at the time when they become smaller than $\eta$. To see this, consider the random finite measure $\rho_{\eta}$ defined by

$$
\left\langle\rho_{\eta}, f\right\rangle:=\sum_{j} x_{\eta, j}^{\alpha} f\left(\frac{x_{\eta, j}}{\eta}\right)
$$

where $f:[0,1] \rightarrow \mathbb{R}_{+}$denotes a generic measurable function and $\left\{x_{\eta, j}\right\}_{j \in \mathbb{N}}$ denotes the set of masses of the fragments of size less than $\eta$ that result from the stopped process. Note that $\rho_{\eta}$ is the measure on $[0,1]$ that puts a mass of $x^{\alpha}$ at $x / \eta$ when $x$ is (the mass of) a fragment in the stopped process.

Corollary 1. We make the same assumptions as in Theorem 1. For every continuous function $f:[0,1] \rightarrow \mathbb{R}_{+}$, we then have

$$
\lim _{\eta \rightarrow 0}\left\langle\rho_{\eta}, f\right\rangle=W_{\infty}\langle\rho, f\rangle \quad \text { in } L^{1}(\mathrm{P})
$$

where $\rho$ is the deterministic probability measure given by

$$
\langle\rho, f\rangle=\frac{1}{m(\alpha)} \int_{0}^{1} f(u)\left(\int_{[0,1]^{\mathbb{N}}} \sum_{n=1}^{\infty} \mathbf{1}_{\left\{u>s_{n}\right\}} s_{n}^{\alpha} v(\mathrm{~d} \mathfrak{s})\right) \frac{\mathrm{d} u}{u} .
$$

Proof. The argument is a variation of that in the proof of Theorem 1. We start from the identity

$$
\left\langle\rho_{\eta}, f\right\rangle=\sum_{i \in \ell} \sum_{n=1}^{\infty} \mathbf{1}_{\left\{\sigma_{i} \leq t<\sigma_{i, n}\right\}} \mathrm{e}^{-\alpha \sigma_{i, n}} f\left(\mathrm{e}^{t-\sigma_{i, n}}\right),
$$

where $\eta=\mathrm{e}^{-t}$ and the notation $\sigma_{i, n}$ refers to the birth time of the $n$th child of the individual $i$.

This motivates the introduction of the process

$$
\phi_{i}(t)= \begin{cases}0 & \text { for } t<0, \\ \sum_{n=1}^{\infty} \mathbf{1}_{\left\{\sigma_{i, n}-\sigma_{i}>t\right\}} \mathrm{e}^{\alpha\left(t-\sigma_{i, n}+\sigma_{i}\right)} f\left(\mathrm{e}^{t-\sigma_{i, n}+\sigma_{i}}\right) & \text { for } t \geq 0\end{cases}
$$


such that

$$
\left\langle\rho_{\eta}, f\right\rangle=\mathrm{e}^{-\alpha t} \sum_{i \in \ell} \phi_{i}\left(t-\sigma_{i}\right)
$$

A simple calculation then gives

$$
\int_{0}^{\infty} \mathrm{e}^{-\alpha t} \mathrm{E}\left(\phi_{\varnothing}(t)\right) \mathrm{d} t=\int_{0}^{1} f(u)\left(\int_{[0,1]^{\mathbb{N}}} \sum_{n=1}^{\infty} \mathbf{1}_{\left\{u>s_{n}\right\}} s_{n}^{\alpha} \nu(\mathrm{d} \mathfrak{s})\right) \frac{\mathrm{d} u}{u},
$$

and the rest of the proof follows that of Theorem 1 .

\section{Continuous framework}

We now turn our attention to the continuous setting, which is probably more appropriate to model the evolution of the processes inside mills and crushers. Indeed, it has been observed in the mining industry that a significant proportion of the particles that pass through the classifying grid after screening are actually of a size much smaller than the mesh of the grid. This suggests that mineral blocks might be subject to a very large number of very small dislocations, which affect only a very small portion of the block.

\subsection{Preliminaries}

The stochastic model that we use is that of homogeneous fragmentation, introduced in [1] and [3]. That is, we consider a Markov process $X(t)=\left(X_{1}(t), X_{2}(t), \ldots\right)$ with values in the infinite simplex of decreasing numerical sequences with sum bounded from above by 1 , i.e.

$$
\S=\left\{\mathfrak{s}=\left(s_{1}, s_{2}, \ldots\right): s_{1} \geq s_{2} \geq \cdots \geq 0, \sum_{1}^{\infty} s_{i} \leq 1\right\} .
$$

We endow $\&$ with the uniform norm; it is readily checked that this turns $\&$ into a compact metric space.

A configuration $\mathfrak{s} \in \delta$ will be called a mass partition; it should be thought of as a ranked family of masses arising from the splitting of a block of unit mass. Note that, for a technical reason, no creation of mass is allowed, i.e. the fragmentation is always conservative or dissipative. Specifically, a portion of the initial mass may be lost during splitting, which corresponds to the situation in which $\sum_{n=1}^{\infty} s_{n}<1$. Small splits correspond to mass partitions $\mathfrak{s}=\left(s_{1}, s_{2}, \ldots\right)$ such that $1-s_{1}$ is small; in other words, in a small split one large fragment is produced and all the remaining ones are small. In particular, the mass partition $(1,0, \ldots)$ must be viewed as a zero element in $\delta$, since it corresponds to a trivial split. It will be convenient to introduce the notation $\delta^{*}=\delta \backslash\{(1,0, \ldots)\}$ for the subspace of nontrivial mass partitions.

We assume that the process satisfies the branching property: different fragments have independent evolutions. Let $\mathrm{P}_{r}$ stand for the law of $X$ starting from $(r, 0, \ldots)$, i.e. such that, at the initial time, there is a single block with mass $r$. Then, for every $t, u \geq 0$ and conditionally on $X(t)=\left(s_{1}, s_{2}, \ldots\right), X(t+u)$ has the same law as the variable obtained by ranking, in decreasing order, the terms of the sequences $X^{(1)}(u), X^{(2)}(u), \ldots$ The latter are independent, random mass partitions with values in $\varnothing$, such that $X^{(n)}(u)$ has the same distribution as $X(u)$ under $\mathrm{P}_{s_{n}}$, for each $n=1,2, \ldots$ We further suppose that the process is homogeneous, in the sense that, for every $r>0$, the distribution of $(r X(t), t \geq 0)$ under $\mathrm{P}_{1}$ is $\mathrm{P}_{r}$. 
It is known that the dynamics of homogeneous fragmentation is characterized by a coefficient of erosion $c \geq 0$ and a dislocation measure $v$ on $\&$ such that $v(\{(1,0, \ldots)\})=0$ and

$$
\int_{\S}\left(1-s_{1}\right) v(\mathrm{~d} \mathfrak{s})<\infty .
$$

Erosion is a deterministic phenomenon (roughly, blocks 'melt' continuously at rate $c$ ) and, for the sake of simplicity, we shall assume in what follows that $c=0$. The dislocation measure specifies the rate at which blocks split: roughly, a block of mass $m$ dislocates into a mass partition $m \mathfrak{s}, \mathfrak{s} \in \mathcal{S}^{*}$, at rate $\nu(\mathrm{d} \mathfrak{s})$.

When the mass of $v$ is finite, the total rate of dislocation of a block is thus $v(8)<\infty$, which means that the block remains unchanged for an exponential time with parameter $v(\&)$, and then splits. In this situation, a logarithmic change of variables transforms the homogeneous fragmentation into a branching random walk in continuous time (see [14]), and the analysis made in the discrete setting can be applied verbatim. The case in which $v(\delta)=\infty$ is thus more interesting, as blocks dislocate immediately: roughly, during any time interval, each block is subject to an infinite number of dislocations, all but finitely many of which are very small.

The cornerstone of the study of homogeneous fragmentation is its Poissonian structure; see [1] and [3]. Specifically, consider a Poisson point process $((\Delta(t), k(t)), t \geq 0)$ with values in $\delta \times \mathbb{N}$ and characteristic measure $v \otimes \sharp$, where $\sharp$ denotes the counting measure on $\mathbb{N}=\{1,2, \ldots\}$. This means that, for every Borel set $B \subset \delta^{*} \times \mathbb{N}$, the process that counts the number of instants $t$ at which $(\Delta(t), k(t)) \in B$, as a function of time, is a Poisson process with intensity $(v \otimes \sharp)(B)$, and that the counting processes corresponding to disjoint Borel sets are independent. There exists a unique pure-jump process $(X(t), t \geq 0)$, with values in $\delta$, that jumps only at those times $t \geq 0$ at which an atom $(\Delta(t), k(t))$ occurs in $\delta^{*} \times \mathbb{N}$. The jump (i.e. the dislocation) induced by such a point can be more precisely described as follows. The sequence $X(t)$ is obtained from $X(t-)$ by replacing its $k(t)$ th term $X_{k(t)}(t-)$ with the sequence $X_{k(t)}(t-) \Delta(t)$, and ranking the terms in decreasing order. For instance, if $X(t-)=\left(\frac{2}{3}, \frac{1}{4}, \frac{1}{12}, 0, \ldots\right), k(t)=2$, and $\Delta(t)=\left(\frac{3}{4}, \frac{1}{4}, 0, \ldots\right)$, then we look at the secondlargest term in the sequence $X(t-)$, namely $\frac{1}{4}$, and split it according to $\Delta(t)$. This produces two fragments of size $\frac{3}{16}$ and $\frac{1}{16}$ and, thus, $X(t)=\left(\frac{2}{3}, \frac{3}{16}, \frac{1}{12}, \frac{1}{16}, 0, \ldots\right)$. Of course, it may happen that $X_{k(t)}(t-)=0$, in which case we have $X(t)=X(t-)$. The process $(X(t), t \geq 0)$ is then a homogeneous fragmentation with no erosion, and dislocation measure $v$.

It will be convenient to introduce the critical value

$$
\beta_{\mathrm{c}}:=\inf \left\{\beta>0: \int_{\S}\left|1-\sum_{n=1}^{\infty} s_{n}^{\beta}\right| v(\mathrm{~d} \mathfrak{s})<\infty\right\},
$$

and then the function $\kappa:\left(\beta_{\mathrm{c}}, \infty\right) \rightarrow \mathbb{R}$ given by

$$
\kappa(\beta):=\int_{\S}\left(1-\sum_{n=1}^{\infty} s_{n}^{\beta}\right) v(\mathrm{~d} \mathfrak{s}) .
$$

Plainly, $\kappa$ is a concave increasing function.

We shall often make use of the fact that, for every $\beta>\beta_{\mathrm{c}}$, the process

$$
\mathrm{e}^{t \kappa(\beta)} \sum_{n=1}^{\infty} X_{n}^{\beta}(t), \quad t \geq 0
$$


is a martingale; see, e.g. Section 2.3 of [5]. (We note that, in the notation of [5], $\beta_{\mathrm{c}}=p+1$ and $\kappa(\beta)=\Phi(\beta-1)$.) This process will henceforth be referred to as the $\beta$-martingale.

In the continuous setting, the Malthusian hypothesis takes the following form:

$$
\text { there exists an } \alpha>\beta_{\mathrm{c}} \text { such that } \kappa(\alpha)=0 \text {. }
$$

Therefore, the process $W_{t}:=\sum_{n=1}^{\infty} X_{n}^{\alpha}(t)$ is a martingale that is always uniformly integrable (see the proof of Theorem 2 of [5] for the argument). We call ( $\left.W_{t}, t \geq 0\right)$ the intrinsic martingale and write $W_{\infty}$ for its terminal value.

Set

$$
m(\alpha):=\kappa^{\prime}(\alpha)=\int_{\S} \sum_{n=1}^{\infty} s_{n}^{\alpha} \log \left(\frac{1}{s_{n}}\right) v(\mathrm{~d} \mathfrak{s}) .
$$

We shall need the following lemma.

Lemma 1. For every $t>0$, we have

$$
\mathrm{E}\left(\sum_{n=1}^{\infty} X_{n}^{\alpha}(t) \log \left(\frac{1}{X_{n}(t)}\right)\right)=\operatorname{tm}(\alpha) .
$$

Proof. Taking the derivative of the $\beta$-martingale with respect to $\beta$ yields the so-called derivative martingale

$$
t \kappa^{\prime}(\beta) \mathrm{e}^{t \kappa(\beta)} \sum_{n=1}^{\infty} X_{n}^{\beta}(t)-\mathrm{e}^{t \kappa(\beta)} \sum_{n=1}^{\infty} X_{n}^{\beta}(t) \log \left(\frac{1}{X_{n}(t)}\right) .
$$

(The martingale property can be checked directly from the Markov property.) Computing the expectation gives

$$
\mathrm{e}^{t \kappa(\beta)} \mathrm{E}\left(\sum_{n=1}^{\infty} X_{n}^{\beta}(t) \log \left(\frac{1}{X_{n}(t)}\right)\right)=t \kappa^{\prime}(\beta) .
$$

Taking $\beta=\alpha$ (meaning that $\kappa(\alpha)=0$ and $\kappa^{\prime}(\alpha)=m(\alpha)$ ), we recover the required formula.

\subsection{Discretization}

Our goal in this section is to derive, by discretization, an extension of Theorem 1 for homogeneous fragmentation. Specifically, fix $a>0$ and consider the discrete skeleton $(X(n a), n \in \mathbb{N})$, which is then a fragmentation chain of the type considered in Section 2 . More precisely, for each individual (i.e. fragment observed at some time $n a$ ), we record not only its size and the sizes of its children, but the entire life career $\omega$ that governs the evolution of this block in the time interval $[n a,(n+1) a]$. To this end, recall the Poissonian construction of homogeneous fragmentation presented at the beginning of this section. The life career of an individual $i$ that corresponds to a block of size $\mathrm{e}^{-\sigma_{i}}$ at, say, time $n a$ is denoted by $\omega_{i}=(\delta(t), \ell(t))_{0<t \leq a}$, where $\delta(t) \in \delta$ and $\ell(t) \in \mathbb{N}$. This means that, for every $t \in(0, a]$, at time $n a+t$ the $\ell(t)$ th largest fragment resulting from this block splits in such a way that the ordered sequence of the resulting size ratios is $\delta(t)$ (of course, there is no split when $\delta(t)=(1,0, \ldots))$.

To define the energy used in an instantaneous dislocation, we consider a measurable cost function $\varphi: s \rightarrow \mathbb{R}$, with $\varphi((1,0, \ldots))=0$. In terms of the discrete skeleton, this leads us to 
introduce a cost function that depends on the life career of individuals. The energy cost for the fragmentation of a block at time na, over $a$ units of time, is thus

$$
\mathrm{e}^{-\beta \sigma_{i}} \varphi_{a}\left(\omega_{i}\right)
$$

where $i$ stands for the individual corresponding to the block, $\omega_{i}$ stands for its life career,

$$
\varphi_{a}\left(\omega_{i}\right)=\sum_{0<t \leq a} s_{\ell(t)}^{\beta}(t-) \varphi(\delta(t)),
$$

and $s_{n}(t) \mathrm{e}^{-\sigma_{i}}$ is the size of the $n$th largest fragment obtained from the block at time $n a+t$. In the special case of a potential energy, i.e. when $\varphi(\mathfrak{s})=\sum_{n=1}^{\infty} s_{n}^{\beta}-1, \varphi_{a}\left(\omega_{i}\right)$ only depends on the sizes of the children of the individual $i$, but in general $\varphi_{a}\left(\omega_{i}\right)$ depends on the whole life career.

We start by computing the mean of the energy for the ancestor $\varnothing$, i.e. the energy spent in fragmenting the initial block of unit size during the time interval $[0, a]$ :

$$
\varphi_{a}\left(\omega_{\varnothing}\right)=\sum_{0<t \leq a} X_{k(t)}^{\beta}(t-) \varphi(\Delta(t)) .
$$

Lemma 2. Suppose that $\beta>\beta_{\mathrm{c}}$. Then, for every $a>0$, we have

$$
\mathrm{E}\left(\varphi_{a}\left(\omega_{\varnothing}\right)\right)=\frac{1-\mathrm{e}^{-a \kappa(\beta)}}{\kappa(\beta)} \int_{\delta} \varphi(\mathfrak{s}) v(\mathrm{~d} \mathfrak{s}) .
$$

Proof. The compensation formula for the Poisson point process $(\Delta(u), k(u))_{u \geq 0}$ gives

$$
\mathrm{E}\left(\sum_{0<t \leq a} X_{k(t)}^{\beta}(t-) \varphi(\Delta(t))\right)=\mathrm{E}\left(\int_{0}^{a} \sum_{n=1}^{\infty} X_{n}^{\beta}(t) \mathrm{d} t\right) \int_{\S} \varphi(\mathfrak{s}) v(\mathrm{~d} \mathfrak{s}) .
$$

Since the mean of the $\beta$-martingale is 1 , we have $\mathrm{E}\left(\sum_{n=1}^{\infty} X_{n}^{\beta}(t)\right)=\mathrm{e}^{-t \kappa(\beta)}$ and the claim follows.

We next fix $\eta>0$ and write $\mathcal{E}(\eta)$ for the energy required to reduce a block of unit size to fragments of size less than $\eta$, using the cost function $\varphi$; that is,

$$
\mathcal{E}(\eta)=\sum_{t \geq 0} \mathbf{1}_{\left\{X_{k(t)}(t-) \geq \eta\right\}} X_{k(t)}^{\beta}(t-) \varphi(\Delta(t)) .
$$

We can now state the following extension of Theorem 1 to the continuous setting.

Theorem 2. Suppose that $\int_{f}|\varphi(\mathfrak{s})| v(\mathrm{~d} \mathfrak{s})<\infty$ and $\beta \in\left(\beta_{\mathrm{c}}, \alpha\right)$. We then have

$$
\lim _{\eta \rightarrow 0} \eta^{\alpha-\beta} \mathcal{E}(\eta)=\frac{W_{\infty}}{(\alpha-\beta) m(\alpha)} \int_{\S} \varphi(\mathfrak{s}) v(\mathrm{~d} \mathfrak{s}) \text { in } L^{1}(\mathrm{P}) .
$$

Before proving Theorem 2, we shall first establish a technical lemma.

Lemma 3. For each $\eta>0$, denote by $\left\{x_{\eta, n}\right\}_{n \in \mathbb{N}}$ the terminal state in the fragmentation process if we freeze each fragment at the instant its size becomes less than $\eta$. For every $\beta \in\left(\beta_{\mathrm{c}}, \alpha\right)$, there then exists a finite constant $c$, which does not depend $\eta$, such that

$$
\mathrm{E}\left(\sum_{n=1}^{\infty} x_{\eta, n}^{\beta}\right) \leq c \eta^{\beta-\alpha} .
$$


Proof. The computation relies on the tagged fragment. Specifically, we shall use an interval representation of the fragmentation (see [4]). That is, we identify the initial block with the unit interval $(0,1)$ and work with a Markovian family $\theta=(\theta(t), t \geq 0)$ of random open subsets of $(0,1)$, nested in the sense that $\theta(s) \subseteq \theta(t)$ for $s \geq t$. The state of the fragmentation at time $t$ is given by the ranked sequence of the lengths of the intervals comprising the components of $\theta(t)$.

Now define, for every $\eta>0$ and $u \in(0,1)$,

$$
T_{\eta}(u):=\inf \left\{t \geq 0:\left|I_{u}(t)\right|<\eta\right\},
$$

where $\left|I_{u}(t)\right|$ denotes the length of the component of $\theta(t)$ containing $u$ (with the convention that $\left|I_{u}(t)\right|=0$ if $\left.u \notin \theta(t)\right)$. We have the identity

$$
\sum_{n=1}^{\infty} x_{\eta, n}^{\beta}=\int_{0}^{1}\left|I_{u}\left(T_{\eta}(u)\right)\right|^{\beta-1} \mathrm{~d} u .
$$

This expression motivates us to introduce a random variable $U$, uniformly distributed on $(0,1)$ and independent of the interval fragmentation $\theta$, and to set

$$
\chi(t):=\left|I_{U}(t)\right|, \quad t \geq 0 .
$$

Hence, $\chi=(\chi(t), t \geq 0)$ is the process of the size of the component of $\theta(t)$ containing a point that has been randomly tagged. If we set

$$
T_{\eta}:=T_{\eta}(U)=\inf \{t \geq 0: \chi(t)<\eta\},
$$

then we have the identity

$$
\mathrm{E}\left(\sum_{n=1}^{\infty} x_{\eta, n}^{\beta}\right)=\mathrm{E}\left(\chi\left(T_{\eta}\right)^{\beta-1}\right) .
$$

It is known, from Theorem 3 of [3], that the process $\xi(t):=-\log \chi(t)$ is a subordinator, i.e. an increasing process with values in $[0, \infty]$ and stationary, independent increments. More precisely, for every $q>\beta_{\mathrm{c}}-1$ and $t \geq 0$, we have

$$
\mathrm{E}(\exp (-q \xi(t)))=\mathrm{E}\left(\chi(t)^{q}\right)=\exp (-t \Phi(q)),
$$

where $\Phi(q)=\kappa(q+1)$ is the so-called Laplace exponent and, on the left-hand side, we have used the convention $\mathrm{e}^{-\infty}=0$.

Because $\Phi(\alpha-1)=0$, the process $\exp (-(\alpha-1) \xi(t))$ is a nonnegative martingale, which we use to tilt the probability; that is, we introduce the probability measure

$$
\mathrm{d} \tilde{\mathrm{P}}_{\mid \mathscr{F}_{t}}=\exp (-(\alpha-1) \xi(t)) \mathrm{dP} \mathscr{F}_{\mid},
$$

where $\left(\mathcal{F}_{t}\right)_{t \geq 0}$ denotes the natural filtration of $\xi$. It is well known that, under $\tilde{\mathrm{P}}, \xi$ is a subordinator with Laplace exponent $\tilde{\Phi}(q)=\Phi(q+\alpha-1)$.

Putting the pieces together, we see that we must bound

$$
\mathrm{E}\left(\sum_{n=1}^{\infty} x_{\eta, n}^{\beta}\right)=\tilde{\mathrm{E}}\left(\exp \left((\alpha-\beta) \xi\left(T_{\eta}\right)\right)\right)
$$


to this end, note that

$$
T_{\eta}=\inf \{t \geq 0: \xi(t)>-\log \eta\}
$$

is the first passage time of the subordinator $\xi$ above the level $-\log \eta$. According to Lemma 1.10 of [2], the distribution of $\xi\left(T_{\eta}\right)$ under $\tilde{\mathrm{P}}$ is given by

$$
\tilde{\mathrm{P}}\left(\xi\left(T_{\eta}\right) \in \mathrm{d} z\right)=\int_{[0,-\log \eta]} \mathbf{1}_{\{z>-\log \eta\}} \tilde{\Pi}(\mathrm{d} z-y) \mathrm{d} \tilde{u}(y),
$$

where $\tilde{U}$ and $\tilde{\Pi}$ are respectively the renewal function and the Lévy measure of $\xi$ under $\tilde{P}$. Using the bound $\eta^{\alpha-\beta} \mathrm{e}^{(\alpha-\beta) z} \leq \mathrm{e}^{(\alpha-\beta) x}$ for $x=z-y, y<-\log \eta$, and $\beta<\alpha$, we obtain

$$
\begin{aligned}
& \eta^{\alpha-\beta} \tilde{\mathrm{E}}\left(\exp \left((\alpha-\beta) \xi\left(T_{\eta}\right)\right), \xi\left(T_{\eta}\right) \geq 1-\log \eta\right) \\
& \quad \leq \int_{[1, \infty)} \mathrm{e}^{(\alpha-\beta) x}\left(\tilde{u}(-\log \eta)-\tilde{u}\left((-\log \eta-x)^{+}\right)\right) \tilde{\Pi}(\mathrm{d} x) \\
& \quad \leq \int_{[1, \infty)} \mathrm{e}^{(\alpha-\beta) x} \tilde{u}(x) \tilde{\Pi}(\mathrm{d} x),
\end{aligned}
$$

where the last line stems from the subadditivity of the renewal function (see [2, p. 10]). We now need only to check the finiteness of the right-hand side of this inequality.

Since $\tilde{\Phi}(0)=0$ and $\tilde{\Phi}^{\prime}(0)=\kappa^{\prime}(\alpha)<\infty$, the subordinator $\xi$ has finite mean under $\tilde{\mathrm{P}}$, given by $\tilde{\mathrm{E}}\left(\xi_{1}\right)=\kappa^{\prime}(\alpha)$, and the elementary renewal theorem ensures that $\tilde{U}(x) \sim x / \kappa^{\prime}(\alpha)$ as $x \rightarrow \infty$. Moreover, for every $q>\beta_{\mathrm{c}}-\alpha$, we know, from the Lévy-Khinchin formula, that

$$
\int_{(0, \infty)}\left(1-\mathrm{e}^{-q x}\right) \tilde{\Pi}(\mathrm{d} x)=\tilde{\Phi}(q)>-\infty
$$

and, since $\beta \in\left(\beta_{\mathrm{c}}, \alpha\right)$, we deduce that

$$
\int_{[1, \infty)} \mathrm{e}^{(\alpha-\beta) x} \tilde{u}(x) \tilde{\Pi}(\mathrm{d} x)<\infty .
$$

This completes the proof of the lemma.

We can now tackle the proof of Theorem 2 .

Proof of Theorem 2. Without loss of generality, we may assume that $\varphi \geq 0$. We write $\varepsilon_{a}(\eta)$ for the energy computed for the discrete skeleton and the cost function $\varphi_{a}$ :

$$
\mathcal{E}_{a}(\eta)=\sum_{i \in \ell} \mathbf{1}_{\left\{\sigma_{i} \leq-\log \eta\right\}} \mathrm{e}^{-\beta \sigma_{i}} \varphi_{a}\left(\omega_{i}\right)
$$

Clearly, $\mathscr{E}_{a}(\eta)$ is an upper bound for $\mathscr{E}(\eta)$ since the former quantity corresponds to the energy spent in the homogeneous fragmentation process when fragments of size less than $\eta$ are frozen at discrete times of the form $n a, n \in \mathbb{N}$, rather than instantaneously.

We need to estimate the overcost $\varepsilon_{a}(\eta)-\mathcal{E}(\eta)$, i.e. the error induced by the discretization. Clearly, if $\left\{x_{\eta, n}\right\}_{n \in \mathbb{N}}$ denotes the terminal state of the process if we freeze each fragment at the instant its size becomes less than $\eta$ in the fragmentation process, the overcost can be bounded from above by

$$
\sum_{n=1}^{\infty} \mathcal{E}\left(\eta, x_{\eta, n}, a\right)
$$


where $\mathcal{E}(\eta, x, a)$ denotes the energy cost for an initial block of size $x$ and an interval of duration $a$ in the fragmentation process. By homogeneity and Lemma 2, the mean overcost can thus be bounded from above by

$$
\begin{aligned}
\mathrm{E}\left(\sum_{n=1}^{\infty} \mathcal{E}\left(\eta, x_{\eta, n}, a\right)\right) & =\frac{1-\mathrm{e}^{-a \kappa(\beta)}}{\kappa(\beta)} \int_{\S} \varphi(\mathfrak{s}) \nu(\mathrm{d} \mathfrak{s}) \mathrm{E}\left(\sum_{n=1}^{\infty} x_{\eta, n}^{\beta}\right) \\
& \leq \eta^{\beta-\alpha} \frac{c\left(1-\mathrm{e}^{-a \kappa(\beta)}\right)}{\kappa(\beta)} \int_{\S} \varphi(\mathfrak{s}) \nu(\mathrm{d} \mathfrak{s}),
\end{aligned}
$$

where the inequality stems from Lemma 3. Putting the pieces together, we have shown, on the one hand, that

$$
\lim _{a \rightarrow 0} \eta^{\alpha-\beta} \mathrm{E}\left(\left|E(\eta)-\mathcal{E}_{a}(\eta)\right|\right)=0, \quad \text { uniformly in } \eta .
$$

On the other hand, the Malthusian hypothesis and the $L \log L$ condition are clearly satisfied for the discrete skeleton, which allows us to apply the results of the preceding section (see Remark 1(c), above). From Lemmas 1 and 2, we obtain

$$
\lim _{\eta \rightarrow 0} \eta^{\alpha-\beta} \mathcal{E}_{a}(\eta)=\frac{W_{\infty}\left(1-\mathrm{e}^{-a \kappa(\beta)}\right)}{(\alpha-\beta) \kappa(\beta) a m(\alpha)} \int_{\delta} \varphi(\mathfrak{s}) v(\mathrm{~d} \mathfrak{s}) \quad \text { in } L^{1}(\mathrm{P}) .
$$

Since $\left(1-\mathrm{e}^{-a \kappa(\beta)}\right) / a \kappa(\beta)$ tends to 1 as $a \rightarrow 0$, this completes the proof of the theorem.

\subsection{The conservative case}

If we compare the discrete and the continuous settings, we see that the hypotheses of Theorem 2 are more restrictive than we might expect, as we can only deal with parameters $\beta$ greater than the critical value $\beta_{\mathrm{c}}$. This restriction stems from the approach via discretization: fragments are frozen at the first discrete time of the form $n a$ at which they become smaller than $\eta$, rather than instantaneously. This implies that the fragmentation process keeps running for some time with blocks of size less than $\eta$, and it turns out that the energy cost for this has a predominant contribution when $\beta \leq \beta_{\mathrm{c}}$.

Our purpose in this section is to provide a direct method of estimating the energy that circumvents this problem. For the sake of simplicity, we shall assume here that the fragmentation is conservative, i.e. that

$$
v\left(\left\{\mathfrak{s} \in \&: \sum_{n=1}^{\infty} s_{n} \neq 1\right\}\right)=0 .
$$

In particular, the Malthusian parameter is $\alpha=1$ and the intrinsic martingale is trivial. We make the following claim.

Theorem 3. Suppose that the homogeneous fragmentation is conservative, that the cost function $\varphi$ belongs to $L^{1}(\mathrm{~d} v) \cap L^{2}(\mathrm{~d} v)$, and that $\beta<1$. We then have

$$
\lim _{\eta \rightarrow 0} \eta^{1-\beta} \mathcal{E}(\eta)=\frac{1}{(1-\beta) m(1)} \int_{\S} \varphi(\mathfrak{s}) v(\mathrm{~d} \mathfrak{s}) \text { in } L^{2}(\mathrm{P}) .
$$

Our approach is based on moment calculations, which are performed using tagged fragments, in the spirit of [5]. The framework is the same as in the proof of Lemma 3, i.e. we work with a Markovian family $(\theta(t), t \geq 0)$ of nested random open subsets of $(0,1)$, and the state of the fragmentation at time $t$ is given by the ranked sequence of the lengths of the intervals comprising 
the components of $\theta(t)$; note that our assumption that the fragmentation is conservative implies that $\theta(t)$ has full Lebesgue measure. Let $U$ be a uniform random variable, independent of $(\theta(t), t \geq 0)$. For each $t \geq 0$, we denote by $\chi(t)$ the length of the component of $\theta(t)$ that contains $U$, and denote by $\chi=(\chi(t), t \geq 0)$ the process of the tagged fragment. The latter provides a powerful tool for computing the first moments of additive functionals of the fragmentation. In particular, the renewal theorem for subordinators yields the following estimate.

Lemma 4. Under the same assumptions as in Theorem 3, we have

$$
\lim _{\eta \rightarrow 0} \eta^{1-\beta} \mathrm{E}(\mathcal{E}(\eta))=\frac{1}{(1-\beta) m(1)} \int_{\S} \varphi(\mathfrak{s}) \nu(\mathrm{d} \mathfrak{s}) .
$$

Proof. Introduce, for every $\eta>0$, the quantity

$$
\tilde{\mathcal{E}}(\eta)=\sum_{t \geq 0} \chi^{\beta-1}(t-) \mathbf{1}_{\{\chi(t-) \geq \eta, k(t)=n(t-)\}} \varphi(\Delta(t)),
$$

where $n(t)$ stands for the index of the tagged fragment at time $t$, i.e. $X_{n(t)}(t)=\chi(t)$. The characteristic function in the sum is 1 if and only if the tagged fragment both splits at time $t$ and has size no less than $\eta$ prior to the split. Note that $\tilde{\mathcal{E}}(\eta)$ can be viewed as the ' $(\beta-1)$ energy' required to reduce the tagged fragment alone to a size less than $\eta$ (in the sense that the exponent $\beta$ in the definition of the energy cost is replaced by $\beta-1$ ). Since, at each time $t \geq 0$, the tagged fragment is a size-biased sample taken from the fragments at time $t$, i.e. $\mathrm{P}(n(t)=\ell \mid X(t))=X_{\ell}(t)$, we see that the conditional expectation of $\tilde{\mathcal{E}}(\eta)$, given the $\sigma$-field generated by the sole fragmentation process (ignoring the uniform random variable $U$ ), is precisely $\mathscr{E}(\eta)$, and, in particular,

$$
\mathrm{E}(\tilde{\mathscr{E}}(\eta))=\mathrm{E}(\mathcal{E}(\eta))
$$

Thus, we must estimate

$$
\mathrm{E}\left(\sum_{t \geq 0} \chi^{\beta-1}(t-) \mathbf{1}_{\{\chi(t-) \geq \eta, k(t)=n(t-)\}} \varphi(\Delta(t))\right) .
$$

To this end, we use the compensation formula for Poisson point processes. We find that

$$
\mathrm{E}(\mathcal{E}(\eta))=\mathrm{E}\left(\int_{0}^{\infty} \chi^{\beta-1}(t) \mathbf{1}_{\{\chi(t) \geq \eta\}} \mathrm{d} t\right) \int_{\mathcal{S}} \varphi(\mathfrak{s}) v(\mathrm{~d} \mathfrak{s}) .
$$

Next, recall from Theorem 3 of [3] that the process $\xi(t):=-\log \chi(t)$ is a subordinator; that is, an increasing process with stationary, independent increments. More precisely, its Laplace exponent

$$
\Phi(q)=-\log \mathrm{E}(\exp (-q \xi(1)))=-\log \mathrm{E}\left(\chi(1)^{q}\right)
$$

is given by $\Phi(q)=\kappa(q+1)$. Hence, if $\mathcal{U}$ denotes the renewal measure of $\xi$, we have

$$
\mathrm{E}\left(\int_{0}^{\infty} \chi^{\beta-1}(t) \mathbf{1}_{\{\chi(t) \geq \eta\}} \mathrm{d} t\right)=\int_{0}^{\log 1 / \eta} \mathrm{e}^{(1-\beta) y} u(\mathrm{~d} y) .
$$


As the subordinator $\xi$ has mean $\Phi^{\prime}(0)=\kappa^{\prime}(1)=m(1)$, it now follows, from the renewal theorem for subordinators, that the quantity above is asymptotically equivalent to

$$
\frac{\eta^{\beta-1}}{m(1)} \int_{0}^{\infty} \mathrm{e}^{-(1-\beta) y} \mathrm{~d} y
$$

as $\eta \rightarrow 0$. This establishes our claim.

Now, to complete the proof of Theorem 3, we need second-moment estimates. To find these, we are led, by the technique of the proof of Lemma 4, to introduce a second independently tagged fragment $\chi^{\prime}=\left(\chi^{\prime}(t), t \geq 0\right)$, where $\chi^{\prime}(t)$ is the length of the component of $\theta(t)$ that contains $U^{\prime}$, another independent uniform variable. In this notation - with primes referring to the second tagged fragment - we see from the same argument, based on size-biased sampling, that

$$
\mathrm{E}\left(\tilde{\mathcal{E}}(\eta) \tilde{\mathcal{E}}^{\prime}(\eta)\right)=\mathrm{E}\left(\mathcal{E}(\eta)^{2}\right),
$$

where

$$
\tilde{\mathcal{E}}^{\prime}(\eta)=\sum_{t \geq 0}\left(\chi^{\prime}\right)^{\beta-1}(t-) \mathbf{1}_{\left\{\chi^{\prime}(t-) \geq \eta, k(t)=n^{\prime}(t-)\right\}} \varphi(\Delta(t)),
$$

and $n^{\prime}(t)$ denotes the index of the second tagged fragment at time $t$. The proof of Theorem 3 will be complete if we can establish the following lemma.

Lemma 5. Under the same assumptions as in Theorem 3, we have

$$
\lim _{\eta \rightarrow 0} \eta^{2-2 \beta} \mathrm{E}\left(\mathcal{E}(\eta)^{2}\right)=\left(\frac{1}{(1-\beta) m(1)} \int_{\S} \varphi(\mathfrak{s}) v(\mathrm{~d} \mathfrak{s})\right)^{2} .
$$

Proof. For the sake of simplicity, we shall focus on the case in which $\varphi \geq 0$, which results in no loss of generality. Let us denote by $T$ the first instant $t$ at which the two tagged points $U$ and $U^{\prime}$ belong to two different components of $\theta(t)$. Clearly, $T$ is a stopping time, so the branching and self-similarity properties enable us to express $\tilde{\mathscr{E}}(\eta)$ and $\tilde{\mathscr{E}}^{\prime}(\eta)$ in the form

$$
\tilde{\mathscr{E}}(\eta)=A+x^{\beta-1} B, \quad \tilde{\mathcal{E}}^{\prime}(\eta)=A+\left(x^{\prime}\right)^{\beta-1} B^{\prime},
$$

where

$$
A=\sum_{t \leq T} \chi^{\beta-1}(t-) \mathbf{1}_{\{\chi(t-) \geq \eta, k(t)=n(t-)\}} \varphi(\Delta(t)),
$$

$x=\chi(T), x^{\prime}=\chi^{\prime}(T)$, and, conditionally on $\left(A, x, x^{\prime}\right), B$ and $B^{\prime}$ are two independent variables distributed as $\mathscr{E}(\eta / x)$ and $\mathscr{E}\left(\eta / x^{\prime}\right)$, respectively. (For the sake of notational simplicity, we have not indicated the dependence of $A, B$, and $B^{\prime}$ on $\eta$.) In particular, Lemma 4 yields

$$
\lim _{\eta \rightarrow 0} \eta^{2-2 \beta} \mathrm{E}\left(x^{\beta-1}\left(x^{\prime}\right)^{\beta-1} B B^{\prime}\right)=\left(\frac{1}{(1-\beta) m(1)} \int_{\S} \varphi(\mathfrak{s}) v(\mathrm{~d} \mathfrak{s})\right)^{2}
$$

and

$$
\eta^{1-\beta} \mathrm{E}\left(x^{\beta-1} B+\left(x^{\prime}\right)^{\beta-1} B^{\prime} \mid A, x, x^{\prime}\right) \leq c,
$$

for some finite constant $c$.

Next, by the compensation formula for Poisson point processes, we have

$$
\mathrm{E}(A)=\mathrm{E}\left(\int_{0}^{\infty} \chi^{\beta-1}(t) \mathbf{1}_{\{\chi(t) \geq \eta\}} \mathbf{1}_{\{t \leq T\}} \mathrm{d} t\right) \int_{\S} \varphi(\mathfrak{s}) v(\mathrm{~d} \mathfrak{s}) .
$$


Observe that the event $\{t \leq T\}$ occurs if and only if $U^{\prime}$ belongs to the component of $\theta(t)$ with length $\chi(t)$ that contains $U$. Moreover, $\chi(t)$ only depends on $U$ and $\theta(t)$, while $U^{\prime}$ is independent of the latter and has the uniform distribution. It follows that

$$
\mathrm{E}\left(\chi^{\beta-1}(t) \mathbf{1}_{\{\chi(t) \geq \eta\}} \mathbf{1}_{\{t \leq T\}}\right)=\mathrm{E}\left(\chi^{\beta}(t) \mathbf{1}_{\{\chi(t) \geq \eta\}}\right) .
$$

Recall, from the proof of Lemma 4, that the tagged fragment process $\chi$ is the exponential of a subordinator $\xi$ with Laplace exponent $\Phi$. Hence,

$$
\mathrm{E}\left(\chi^{\beta}(t) \mathbf{1}_{\{\chi(t) \geq \eta\}}\right) \leq \mathrm{E}\left(\mathrm{e}^{-\beta \xi(t)}\right)=\mathrm{e}^{-t \Phi(\beta)}
$$

and we conclude that

$$
\mathrm{E}(A)<\frac{1}{\Phi(\beta)} \int_{\S} \varphi(\mathfrak{s}) v(\mathrm{~d} \mathfrak{s})
$$

As a consequence of (2),

$$
\lim _{\eta \rightarrow 0} \eta^{2-2 \beta} \mathrm{E}\left(A\left(x^{\beta-1} B+\left(x^{\prime}\right)^{\beta-1} B^{\prime}\right)\right)=0 .
$$

We now turn our attention to the mean of $A^{2}$, a variable that we express in the form $C+2 D$, with

$$
\begin{aligned}
C= & \sum_{0<t \leq T} \chi^{2 \beta-2}(t-) \mathbf{1}_{\{\chi(t-) \geq \eta, k(t)=n(t-)\}} \varphi(\Delta(t))^{2} \\
D= & \sum_{0<t \leq T} \chi^{\beta-1}(t-) \mathbf{1}_{\{\chi(t-) \geq \eta, k(t)=n(t-)\}} \varphi(\Delta(t)) \\
& \times \sum_{t<u \leq T} \chi^{\beta-1}(u-) \mathbf{1}_{\{\chi(u-) \geq \eta, k(u)=n(u-)\}} \varphi(\Delta(u)) .
\end{aligned}
$$

We compute $\mathrm{E}(C)$ using the compensation formula, to obtain

$$
\begin{aligned}
\mathrm{E}(C) & =\mathrm{E}\left(\int_{0}^{\infty} \chi^{2 \beta-2}(t) \mathbf{1}_{\{\chi(t) \geq \eta, t<T\}} \mathrm{d} t\right) \int_{\S} \varphi(\mathfrak{s})^{2} v(\mathrm{~d} \mathfrak{s}) \\
& =\mathrm{E}\left(\int_{0}^{\infty} \chi^{2 \beta-1}(t) \mathbf{1}_{\{\chi(t) \geq \eta\}} \mathrm{d} t\right) \int_{\delta} \varphi(\mathfrak{s})^{2} v(\mathrm{~d} \mathfrak{s}),
\end{aligned}
$$

where the last equality follows from (3) with $2 \beta-1$ in place of $\beta$. Now recall (1): when $\eta \rightarrow 0$, this quantity remains bounded for $\beta>\frac{1}{2}$, and is of order $\log 1 / \eta$ for $\beta=\frac{1}{2}$ (by the elementary renewal theorem) and of order $\eta^{2 \beta-1}$ for $\beta<\frac{1}{2}$ (by the renewal theorem).

Finally, we compute $\mathrm{E}(D)$ by conditioning at those times $t$ at which the tagged fragment splits. By an application of the regenerative property of Poisson point processes at times when atoms appear, and Lemma 4, we find that

$$
\mathrm{E}(D) \leq a \eta^{\beta-1} \mathrm{E}(A),
$$

for some finite constant $a$. Recall, from (4), that $\mathrm{E}(A)$ remains bounded as $\eta \rightarrow 0$. Putting the pieces together, we have shown that $\lim _{\eta \rightarrow 0} \eta^{2-2 \beta} \mathrm{E}\left(A^{2}\right)=0$, which completes the proof of the statement. 
We now conclude this section with a continuous analogue of Corollary 1 , which specifies the asymptotics of the empirical distribution of the terminal state of the process when fragments are frozen at the instant they become smaller than $\eta$. Introduce the random probability measure $\rho_{\eta}$ defined by

$$
\left\langle\rho_{\eta}, f\right\rangle:=\sum_{j} x_{\eta, j} f\left(\frac{x_{\eta, j}}{\eta}\right),
$$

where $f:[0,1] \rightarrow \mathbb{R}_{+}$denotes a generic measurable function and $\left\{x_{\eta, j}\right\}_{j \in \mathbb{N}}$ stands for the set of fragments of size less than $\eta$ that result from the process.

Corollary 2. We make the same assumptions as in Theorem 3. For every continuous function $f:[0,1] \rightarrow \mathbb{R}_{+}$, we then have

$$
\lim _{\eta \rightarrow 0}\left\langle\rho_{\eta}, f\right\rangle=\langle\rho, f\rangle \quad \text { in } L^{2}(\mathrm{P})
$$

where $\rho$ is the deterministic probability measure given by

$$
\langle\rho, f\rangle=\frac{1}{m(1)} \int_{0}^{1} f(u)\left(\int_{\S} \sum_{n=1}^{\infty} \mathbf{1}_{\left\{u>s_{n}\right\}} s_{n} v(\mathrm{~d} \mathfrak{s})\right) \frac{\mathrm{d} u}{u} .
$$

The proof follows from the same type of calculations as did Theorem 3; the details are therefore left to the reader. Alternatively, we may establish Corollary 2 by first considering the case in which $f(x)=x^{\beta-1}$ is a power function:

$$
\left\langle\rho_{\eta}, f\right\rangle:=\eta^{1-\beta} \sum_{j} x_{\eta, j}^{\beta} .
$$

As observed in the introduction, we can express the right-hand side in terms of the potential energy; that is, for the choice $\varphi(\mathfrak{s})=\sum_{n=1}^{\infty} s_{n}^{\beta}-1$. We obtain

$$
\left\langle\rho_{\eta}, f\right\rangle:=\eta^{1-\beta}(\mathscr{E}(\eta)+1) \text {. }
$$

Then Theorem 3 clearly implies Corollary 2 in the case that $f$ is a power function, and the general case follows by standard arguments.

\section{Conclusions}

In any industry, one strives to minimize the production cost. If the model developed in this work is applied to the mining industry, then the dislocation measure $v$, the cost function $\varphi$, the exponent $\beta$, and the Malthusian parameter $\alpha$ will depend essentially on the quality of the mineral extract and the properties of the crushers, grinders, and mills. These data are given and can hardly be modified: the quality of the mineral extract is given by the geological and geophysical characteristics of the pit section being exploited, while crushers are heavy and expensive items of equipment that receive prolonged use and are seldom replaced. However, there is some flexibility in the choice of the blasting operation, the size setting of the crushers, and the mesh of the grid used for screening. Roughly, our results show that the energy cost behaves like a certain power function of the mesh size of the grid (with a negative exponent $\beta-\alpha$ ). A larger mesh results in a smaller fragmentation cost, but if the process produces fragments of larger size, the next step (physicochemical processing) will be less efficient. Thus, one should also estimate the cost of the physicochemical processing as a function of the size of the fragments, and then optimize the procedures simultaneously. 


\section{Acknowledgements}

We would like to express our gratitude to Felipe Mujica (IM2, Codelco) for very useful discussions and comments. We also would like to thank an anonymous referee for pointing out an error in an earlier version of this work. J. Bertoin acknowledges support from Nucleus Millennium Information and Randomness P01-005 for his visit to CMM-DIM in Santiago. S. Martínez' research is also supported by Nucleus Millennium Information and Randomness P01-005.

\section{References}

[1] Berestycki, J. (2002). Ranked fragmentations. ESAIM, Prob. Statist. 6, 157-176.

[2] Bertoin, J. (1999). Subordinators: examples and applications. In Lectures on Probability Theory and Statistics (St-Flour, 1997; Lecture Notes Math. 1717), Springer, Berlin, pp. 1-91.

[3] Bertoin, J. (2001). Homogeneous fragmentation processes. Prob. Theory Relat. Fields 121, 301-318.

[4] Bertoin, J. (2002). Self-similar fragmentations. Ann. Inst. H. Poincaré Prob. Statist. 38, 319-340.

[5] Bertoin, J. (2003). The asymptotic behavior of fragmentation processes. J. Europ. Math. Soc. 5, $395-416$.

[6] Biggins, J. D. (1977). Martingale convergence in the branching random walk. J. Appl. Prob. 14, 25-37.

[7] Bond, F. C. (1952). The third theory of conminution. AIME Trans. Vol. 193, 484.

[8] Charles, R. J. (1957). Energy-size reduction relationships in conminution. AIME Trans. Vol. 208, 80-88.

[9] Devoto, D. and Martínez, S. (1998). Truncated Pareto law and oresize distribution of ground rocks. Math. Geology 30, 661-673.

[10] Jagers, P. (1989). General branching processes as Markov fields. Stoch. Process. Appl. 32, 183-212.

[11] Nerman, O. (1981). On the convergence of supercritical general (C-M-J) branching processes. Z. Wahrscheinlichkeitsth. 57, 365-395.

[12] Perrier, E. M. ANd Bird, N. R. (2002). Modelling soil fragmentation: the pore solid fractal approach. Soil Tillage Res. 64, 91-99.

[13] Turcotte, D. L. (1986). Fractals and fragmentation. J. Geophys. Res. 91, 1921-1926.

[14] UchiYAma, K. (1982). Spatial growth of a branching process of particles living in $R^{d}$. Ann. Prob. 10, 896-918.

[15] Walker, W. H., Lewis, W. K., McAdams, W. H. and Gilliland, E. R. (1967). Principles of Chemical Engineering. McGraw-Hill, New York.

[16] Weiss, N. L. (ed.) (1985). SME Mineral Processing Handbook, Vol. 1. Society of Mining Engineers of the American Institute of Mining, Metallurgical, and Petroleum Engineers, New York, pp. 3A-28-3A-55. 